\title{
Controle da qualidade em colpocitologia: visão rápida com campo marcado
}

\section{Cervical cytology quality control: rapid pre-screening with marked field}

Jane Lopes Bonilha'; Cláudia de Fátima Moriel Valença²; Juliana Pignatari Micelli³; Eliane Milhacrix Zanovelo ${ }^{3}$; Janice Silva ${ }^{4}$; Patrícia Maluf Cury ${ }^{4}$

Unitermos
Pré-escrutínio rápido
Colpocitologia
Controle da qualidade

\section{resumo}

Introdução: No Departamento de Patologia e Medicina Legal da Faculdade de Medicina de São José do Rio Preto (FAMERP), adotou-se um sistema de gestão da qualidade (SGQ) segundo as normas brasileiras preconizadas pela International Organization for Standardization (NBR ISO) 9001: 2000. Esse procedimento foi adotado para assegurar a confiabilidade técnica dos exames realizados, em que a qualidade é considerada como julgamento do cliente, ou seja, solução às suas expectativas. Neste estudo, focamos a qualidade dos exames colpocitológicos realizados pelo Departamento. Embora a evolução de lesões do colo do útero para carcinoma invasor seja lenta, o êxito do tratamento depende do seu diagnóstico precoce. Assim, entre as possíveis falhas do exame preventivo, diagnóstico falso-negativo é a que mais agravo causa, pois posterga o início do tratamento. Objetivo: Testar três métodos diferentes, procurando aquele com menor número de diagnósticos falso-negativos. Material e método: Com a mesma equipe examinadora e com os mesmos critérios de diagnóstico, testamos o método 1 (M1), preconizado pelo Ministério da Saúde (MS), que faz revisão aleatória de $10 \%$ dos casos negativos, em 3.500 colpocitologias; o método 2 (M2): revisão rápida (RR), proposto na literatura, que consiste de revisão rápida de $100 \%$ dos casos, após o escrutínio, em 7.373 casos; e o método 3 (M3): visão rápida (VR) com campo marcado, delineado pela pesquisadora, propõe o exame rápido pré-escrutínio de todos os casos, marcando-se um campo em que existam células alteradas, quando visualizadas, em 8.096 citologias ginecológicas. Resultados: Foram encontradas as seguintes porcentagens de diagnósticos falso-negativos: M1: 2,4\%; M2: 1,7\% e M3: 1,2\%. Conclusão: O método M3 foi o melhor, apresentando menor quantidade de diagnósticos falso-negativos. abstract

Introduction: The Pathology and Forensic Medicine Department of the Faculdade de Medicina de São José do Rio Preto, SP had decided to develop a Quality Control System following the NBR ISO 9001: 2000 guidelines. Some procedures were adopted to verify technical reliability for the exams performed, where quality is defined as the clients' judgement, i.e., the solution for the clients' requests and expectations. In this study we have focused on the quality of the cervical cytology performed in the Department. Although the development of cervical lesions through cervical carcinoma takes place slowly, the treatment will have a better chance of success in case of an early diagnosis. Considering all the possible diagnostic mistakes, the false-negative exam is the most dangerous, as it delays the beginning of the treatment. Objective: To test three different methods looking for the one with the smaller number of false-negative results. Material and methods: For the same team of technicians, and with the same diagnostic criteria the three different techniques were tested. Methodology 1 (M1): in accordance to the Brazilian Ministry of National Health, with a random review of $10 \%$ of the false-negative results, in the 3,500 cytologies; Methodology 2 (M2): rapid review (RR), as suggested in literature, consists of a rapid review of 100\% of the smears, after their routine evaluation, and was done in 7,373 cytologies; Methodology 3 (M3): rapid prescreening $(R P)$ with marked field, was proposed by the researcher and consists of a rapid screening of all cases, pointing out a field were there are abnormal cells, and after that the normal screening, in 8,096 cytologies. Results: The three techniques tested showed the following false-negative rates: M1: 2.4\%; M2: 1.7\% and M3: 1.2 \%. Conclusion: The M3 was the best methodology, presenting less false-negative diagnosis. key words Rapid pre-screening Cervical cytology Quality control

\footnotetext{
1. Citopatologista.

2. Bióloga/citotécnica.

3. Residentes em Anatomia Patológica.

4. Patologistas.

Trabalho realizado no Departamento de Patologia e Medicina Legal da Faculdade de Medicina de São José do Rio Preto (FAMERP). Programa de Pós-graduação em Ciências da Saúde (eixo temático: medicina interna), baseado na tese de doutorado intitulada A citopatologia ginecológica: gestão da qualidade.
} 


\section{Introdução}

Em um serviço de citopatologia, a incidência de resultados falso-negativos (FN) e falso-positivos (FP) é preocupante, devido a todas as conseqüências que acarreta para a mulher e também em virtude dos custos com o tratamento médico. Considera-se o diagnóstico colpocitológico como $\mathrm{FN}$ aquele que ocorre em um exame que tem a lesão do colo do útero representada no esfregaço examinado, porém não é identificada e/ou é interpretada equivocadamente como alteração inflamatória, reparativa ou secundária à ação radioterápica. Ao contrário, o diagnóstico FP ocorre em esfregaços em que as alterações inflamatórias, reparativas ou radioterápicas são supervalorizadas e interpretadas como anormalidades pré-malignas ou malignas.

A taxa de diagnósticos $\mathrm{FN}$, segundo dados da literatu$\mathrm{ra}^{(15,16,25,33)}$, apresenta variações de até $68 \%$, fazendo com que alguns serviços percam a credibilidade ou mesmo colocando em dúvida a validade do exame de Papanicolaou na detecção precoce de câncer do colo uterino. As causas mais importantes de resultados FN são erros na coleta, na fixação, no armazenamento e transporte, na identificação do material, na coloração dos esfregaços, bem como no exame microscópico e/ou na interpretação diagnóstica das alterações morfológicas observadas. Alguns autores afirmam que há evidências de que a maioria dos erros ocorre na fase pré-analítica ${ }^{(20)}$.

Existem alguns métodos para garantir a qualidade do diagnóstico. O Ministério da Saúde (MS) recomenda a revisão de $10 \%$ dos casos negativos ${ }^{(24)}$. No entanto, embora possa detectar falhas, $90 \%$ dos exames negativos não são revisados.

Mais recentemente, alguns autores têm recomendado a revisão de $100 \%$ dos casos escrutinados, através de um método denominado revisão rápida $(\mathrm{RR})^{(2)}$. Nele, se reexamina a lâmina toda, com uma objetiva de $10 \mathrm{X}$, em um minuto, observando-se apenas alterações da morfologia celular.

Decidimos testar uma nova maneira, que idealizamos, para controle da qualidade dos diagnósticos colpocitológicos. O processo adotado foi o exame rápido e aleatório de todo o esfregaço (antes do escrutínio), em 100\% dos casos, fazendo-se a marcação de uma célula ou de um grupo de células com alterações morfológicas, quando presentes no esfregaço, sem preocupação com seu possível diagnóstico. Esse método de exame pré-escrutínio rápido, com marcação de células, denominamos de visão rápida (VR) com campo marcado.

\section{Objetivo}

O escopo deste trabalho foi testar três métodos de controle interno da qualidade de diagnóstico dos exames colpocitológicos, para verificar qual apresenta o menor número de diagnósticos FN.

\section{Material e método}

O trabalho foi realizado no Serviço de Anatomia Patológica do Hospital de Base da Faculdade de Medicina de São José do Rio Preto (FAMERP), avaliando colpocitologias de rotina de exames realizados entre 2003 e 2004. Todos os casos foram corados pelo método de Papanicolaou, e a média mensal de exames foi de cerca de 1.300 .

O estudo mediu o número de diagnósticos falso-negativos em três métodos diferentes de controle interno da qualidade dos diagnósticos colpocitológicos, aos quais denominamos de:

- método 1 (M1): preconizado pelo MS;

- método 2 (M2): revisão rápida (RR);

- método 3 (M3): visão rápida (VR) com campo marcado.

O exame microscópico foi realizado pela equipe examinadora do serviço, conforme descrito na Figura 1, variando a rotina segundo o método testado. A equipe conta com uma citopatologista, responsável pelo serviço, uma patologista, uma citotécnica e duas residentes (uma do $2^{\circ}$ ano e outra do $3^{\circ}$ ).

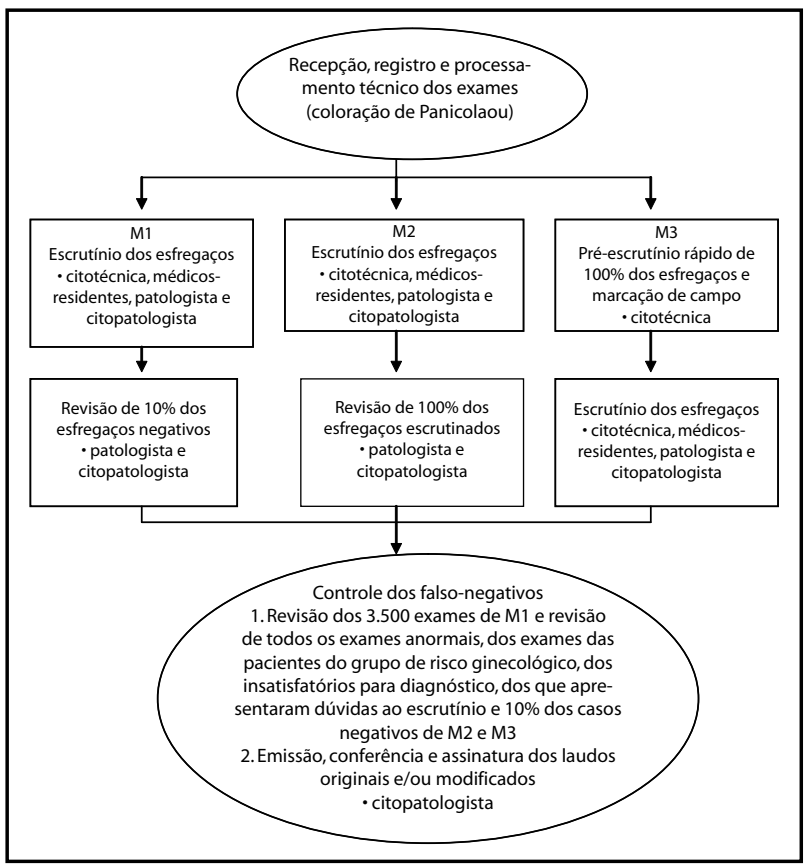


Foram considerados negativos os exames com diagnósticos dentro dos limites de normalidade e/ou com alterações inflamatórias, metaplásicas, reparativas ou radioterápicas. Foram considerados exames alterados ou anormais aqueles com diagnóstico de alterações morfológicas em células escamosas atípicas de significado indeterminado (ASCUS), lesão intra-epitelial de baixo grau (LIEBG) ou lesão intraepitelial de alto grau (LIEAG), carcinoma espinocelular in situ ou invasor, atipias glandulares e adenocarcinoma in situ ou invasor.

No M1, com 3.500 exames selecionados para o estudo, as lâminas foram escrutinadas e apenas 10\% dos casos negativos foram revisados, e todos os casos alterados ou com dúvidas de interpretação das alterações morfológicas. No M2, selecionamos 7.373 exames, onde todos os casos escrutinados foram revistos. Já no método da VR, com 8.096 exames, $100 \%$ dos casos sofreram exame pré-escrutínio rápido, com marcação de um campo com célula(s) alterada(s).

O controle dos diagnósticos nos três métodos foi por reescrutínio de exames pela citopatologista. No M1, os 3.500 casos foram revisados. Nos métodos 2 e 3 , nos quais cada exame já tinha passado por dois exames microscópicos (RR ou VR), foram reexaminados $10 \%$ dos casos negativos, todas as pacientes do grupo de risco ginecológico, todos os casos de material insatisfatório para diagnóstico e todos os casos de escrutínio/RR ou VR/escrutínio com diagnósticos discordantes. Nos casos discordantes, o diagnóstico final foi o da médica citopatologista, que foi considerado padrãoouro, pois muitos dos casos de colpocitologias anormais não apresentavam biópsia de colo do útero concomitante.

Paralelamente, todos os casos com colpocitologia e/ou biópsia de colo uterino alteradas, atualmente, foram encaminhados à reunião de estudo, onde foram revistas também todas as citologias ginecológicas e biópsias de colo do útero: todos os exames anteriores, da mesma paciente, realizados no Serviço, nos últimos cinco anos.

\section{Resultados}

No M1 foram escrutinadas 3.500 colpocitologias, escolhidas aleatoriamente entre os exames registrados em seis meses sorteados em 2003. Foram revisados $10 \%$ dos casos negativos e 178 dos positivos ao escrutínio. Como resultado dessa revisão, os 178 casos positivos ficaram reduzidos a 129, sendo que 49 eram, portanto, falso-positivos. Foram detectados 18 casos falso-negativos entre os 335 selecionados aleatoriamente, para constituírem os $10 \%$ negativos revisados. Em seguida, realizou-se o reescrutínio (revisão rápida em um minuto por caso) para controle dos falso-negativos de todos os 2.987 exames liberados como negativos. Esse reescrutínio mostrou 2.920 casos verdadeiro-negativos e 67 falso-negativos. Os resultados finais estão sintetizados na Tabela 1.

O M2 (RR), no qual ocorreu revisão rápida de todos os casos após o escrutínio de rotina, foi aplicado entre setembro de 2003 e fevereiro de 2004 em 7.373 colpocitologias. Após o escrutínio e a revisão rápida registramos 357 casos anormais, que foram identificados pelo escrutínio. Já a RR do total de casos mostrou 462 verdadeiropositivos, concluindo-se que 105 seriam falso-negativos após o escrutínio.

Para aferir a qualidade dos resultados mencionados anteriormente, fizemos o reescrutínio e constatamos que dos $10 \%$ de casos considerados negativos após escrutínio e revisão rápida, cinco eram, na verdade, anormais e, portanto, FN. Todos os 13 casos insatisfatórios para diagnóstico assim permaneceram e não apresentavam células atípicas identificáveis nos esfregaços. Dos 347 casos discordantes entre o escrutínio e a revisão rápida, verificou-se que 232 eram FP pelo padrão-ouro e 115, falso-negativos (dez casos a mais que ao escrutínio, que totalizara 105 FN). Das 311 pacientes revisadas do grupo de risco para câncer do colo do útero, 306 continuaram com diagnóstico negativo e cinco tiveram resultados considerados falso-negativos. A

\section{Tabela 1 Resultados finais após $100 \%$ de revisäo dos 3.500 casos para controle de falso-negativos}

\begin{tabular}{|c|c|c|c|c|}
\hline \multicolumn{5}{|c|}{ Padrão-ouro } \\
\hline$E$ & & POS & NEG & Total \\
\hline S & POS & 129 (VP) & 49 (FP) & 178 \\
\hline c & NEG & $85(\mathrm{FN})$ & 3.237 (VN) & 3.322 \\
\hline u & Total & 214 & 3.286 & 3.500 \\
\hline
\end{tabular}

Escrut: diagnóstico dos escrutinadores (citotécnica, médicos residentes e patologista); padrão-ouro: diagnóstico da pesquisadora; POS: casos positivos; NEC: casos negativos; VP: número de casos verdadeiro-positivos; FN: número de casos falso-negativos; FP: número de casos falso-positivos; VN: número de casos verdadeiro-negativos. 
Tabela 2 mostra como ficaram esses resultados após as duas fases de exame dos esfregaços e do reescrutínio para controle de falso-negativos.

O exame pré-escrutínio rápido com campo marcado, seguido de escrutínio (M3), foi aplicado entre março e agosto de 2004 em 8.096 colpocitologias registradas.

Nessa amostragem ocorreram 267 casos verdadeiropositivos, identificados pelo escrutínio. Já a visão rápida com campo marcado de todos os casos identificou 358 considerados anormais, sendo 91 exames falso-positivos.

No reescrutínio para controle dos FN foram revistos 1.575 exames. No sorteio de $10 \%$ dos casos considerados negativos ao escrutínio, oito eram, de fato, anormais e, portanto, falso-negativos. Dos 35 casos insatisfatórios para diagnóstico, 34 assim permaneceram e um caso apresentou poucas células atípicas para malignidade, não sendo possível classificá-lo pelos critérios diagnósticos estabelecidos e, conseqüentemente, não pôde ser considerado falso-negativo.

Dos 247 casos discordantes, verificou-se que 185 eram FP pelo padrão-ouro e 62, FN. Das 375 pacientes do grupo de risco revisadas, 351 continuaram com diagnóstico negativo e 24 foram considerados FN. Dessa forma foram detectados 94 casos falso-negativos por esse método, somando um total de 361 casos anormais na amostra examinada, de 8.096 mulheres, conforme apresentado na Tabela 3.

Os resultados do estudo estatístico estão resumidos nas Tabelas 4 e 5.

\section{Tabela 2 rápida}

\section{Resultados finais do escrutínio, revisão rápida e reescrutínio segundo o método 2: revisão}

\begin{tabular}{|c|c|c|c|c|}
\hline \multicolumn{5}{|c|}{ Padrão-ouro } \\
\hline E & & POS & NEG & Total \\
\hline $\mathrm{s}$ & POS & 357 (VP) & 232 (FP) & 589 \\
\hline $\begin{array}{l}c \\
r\end{array}$ & NEG & 125 (FN) & $6.659(\mathrm{VN})$ & 6.784 \\
\hline $\begin{array}{l}\mathrm{u} \\
\mathrm{t}\end{array}$ & Total & 482 & 6.891 & 7.373 \\
\hline
\end{tabular}

Escrut: diagnóstico dos escrutinadores (citotécnica, médicos residentes e patologista); padrão-ouro: diagnóstico da pesquisadora; POS: casos positivos; NEC: casos negativos; VP: número de casos verdadeiro-positivos; FN: número de casos falso-negativos; FP: número de casos falso-positivos; VN: número de casos verdadeiro-negativos.

\section{Tabela 3}

Resultados da visão rápida com campo marcado e escrutínio, conforme 0 método 3: visão

\begin{tabular}{|c|c|c|c|c|}
\hline \multicolumn{5}{|c|}{ Padrão-ouro } \\
\hline$E$ & & POS & NEG & Total \\
\hline S & POS & 267 (VP) & 185 (FP) & 452 \\
\hline $\begin{array}{l}c \\
r\end{array}$ & NEG & 94 (FN) & 7.550 (VN) & 7.644 \\
\hline $\begin{array}{l}\mathrm{u} \\
\mathrm{u}\end{array}$ & Total & 361 & 7.735 & 8.096 \\
\hline
\end{tabular}

\section{Margem de erro dos diagnósticos falso-negativos do teste de Papanicolaou para os três}

\section{Tabela 4}

Método

\section{1}

M 2

M 3
Porcentagem de diagnósticos falso-negativos e IC
$2,4 \%$
IC $95 \%=2$ a 3
$1,7 \%$
IC $95 \%=1,4$ a 2
$1,2 \%$

M1: método 1: preconizado pelo Ministério da Saúde; M2: método 2: revisão rápida; M3: método 3: visão rápida com campo marcado; intervalo de confiança (IC) 95\%: existe 95 em 100 chances de estar correto o número de diagnósticos falso-negativos existentes no método estudado, pois se situa no intervalo entre o limite inferior e o limite superior. 


\section{Tabela 5 Resultado do cálculo de significância das hipóteses nulas formuladas}

\begin{tabular}{lll}
\hline Ho proposta & $p(\%)$ & IC $95 \%$ \\
Ho: M3 - M2 = & $=0,3$ & $-0,16$ a 0,91 \\
Ho: M3 - M1 = & $<0,05$ & $-0,07$ a 1,83 \\
Ho: M2 - M1 = 0 & $=0,7$ & $-0,14$ a 1,32 \\
\hline
\end{tabular}

Ho: hipótese nula; M1: método preconizado pelo Ministério da Saúde; M2: método da revisão rápida; M3: método da visão rápida com campo marcado; p:; IC 95 \%: existe 95 em 100 chances de estar correto o número de diagnósticos falso-negativos existentes no método estudado, pois se situa no intervalo entre o limite inferior e o limite superior calculados.

Foram aventadas e invalidadas as seguintes hipóteses nulas:

- Não existe diferença entre o número de diagnósticos falso-negativos obtidos pelos métodos M3 e M2;

- Não existe diferença entre o número de diagnósticos falso-negativos obtidos pelos métodos M3 e M1;

- Não existe diferença entre o número de diagnósticos falso-negativos obtidos pelos métodos M2 e M1.

Quanto ao tempo médio despendido (em segundos) para o exame microscópico em cada um dos três métodos testados, verificamos que o M1 consumiu 329 segundos por lâmina; o M2, 205; e o M3, 180.

\section{Discussão}

Muitos autores $(6,18,21,27,28,32,35,36)$ salientam que podem ocorrer erros no diagnóstico do teste de Papanicolaou.

Para comprovar a hipótese de que os exames colpocitológicos apresentam resultados falso-negativos e verificar qual a melhor maneira de se obter menos resultados FN na rotina diária, aplicamos três diferentes métodos, sendo que o preconizado pelo MS é um dos mais utilizados pelos laboratórios de patologia, em todo o mundo, para o controle interno da qualidade $\mathrm{e}^{(1,17,19,23)}$.

Este estudo mostra taxas de falso-negativos de 2,4\%, $1,7 \%$ e 1,2\%, respectivamente, em M1, M2 e M3. As taxas de ocorrência de diagnósticos $\mathrm{FN}$ são variáveis, entre os diversos autores consultados ${ }^{(15,16,25,33)}$. Houve autor ${ }^{(9)}$ que encontrou $5 \%$ de $\mathrm{FN}$ em sua amostra. Para outro pesquisador ${ }^{(26)}$, os falso-negativos variaram de $2 \%$ a $28 \%$, enquanto outro pesquisador ${ }^{(34)}$ relatou $1,22 \%$ de falso-negativos e 0,26\% de falso-positivos.

É necessário revisar todos os casos FN e FP, visando identificar as causas dos erros de diagnóstico. Só então será possível saber onde e como agir. Os diagnósticos falsonegativos podem ser por falhas na coleta da amostragem, no rastreamento de anormalidades e na interpretação das alterações morfológicas ${ }^{(30)}$. Para outros autores ${ }^{(13)}$, os erros na coleta do material ocorrem em $62 \%$ dos casos, no escrutínio do esfregaço em $16 \%$ e na interpretação dos achados microscópicos em $22 \%$. Verificamos ainda que se atribuem mais de 50\% dos falso-negativos à amostragem inadequada ${ }^{(14)}$. Portanto, acreditamos que para a correção de falhas de amostragem são necessárias ações de treinamento e/ou educação continuada para o pessoal envolvido na coleta dos esfregaços (fase pré-analítica).

Constatamos em nosso trabalho que em $73,5 \%$ dos exames falso-negativos que tiveram diagnóstico inicial de alterações inflamatórias, existiam raras células anormais (de 3 a 28) por esfregaço. Outros autores ${ }^{(4)}$ também concluíram que diagnósticos falso-negativos se devem a um número quantitativamente menor de células anormais, quando em comparação com os esfregaços verdadeiro-positivos.

Outro fator que afeta o diagnóstico é a sua subjetividade. Há um contrabalanço (trade-off) variável entre o julgamento do padrão-ouro e aquele dos demais componentes da equipe examinadora. O ponto de corte entre o que é negativo e o que é anormal, ou alteração pré-neoplásica/ neoplásica, é determinado arbitrariamente. No teste de Papanicolaou esse ponto de corte consiste em considerar determinadas alterações presentes nas células examinadas como alterações inflamatórias acentuadas ou como anormalidades pré-malignas/malignas.

Para calcular a significância dos nossos resultados quando comparamos os três métodos utilizados neste estudo, aplicamos o teste da distribuição normal bivariada com cálculo do intervalo de confiança (IC), através de um pacote estatístico. Assim, verificamos que o M3 é melhor do que o M2 e do que o M1, e que o M2 é melhor do que o M1. Com a correção de Bonferroni, houve constatação estatística de que o M3 (VR) com campo marcado é o melhor entre os três métodos testados. Pode-se ter um número ainda menor de diagnósticos falso-negativos no $\mathrm{M} 3$, se as lâminas das pacientes do grupo de risco ginecológico, que têm seus critérios definidos no Programa Viva Mulher ${ }^{(24)}$, estiverem identificadas ao fazer o exame pré-escrutínio. 
Verificamos que para alcançar bons resultados, é necessário que se crie um protocolo no serviço laboratorial, definindo os critérios diagnósticos a serem seguidos por toda equipe examinadora, tanto para os exames colpocitológicos como para os anatomopatológicos de colo uterino, e que os mesmos estejam bem treinados, equilibrando o fator subjetividade dos diagnósticos. A correlação clínica/cito-histológica é importante instrumento de ensino e aprendizado e, portanto, de educação continuada em citopatologia ginecológica.

Reuniões de estudo realizadas pela equipe examinadora para revisar sistematicamente e discutir em conjunto, com juízo crítico, os casos anormais, sejam os de biópsias de colo do útero ou os de colpocitologias alteradas, atuais ou anteriores, melhoram a performance dos examinadores.

Por outro lado, a RR e a VR possibilitam detectar examinadores com dificuldades e/ou deficiências no diagnóstico, quando eles geram diagnósticos discordantes daqueles do padrão-ouro. Treinamentos para esses examinadores com deficiências devem ser repetidos sempre que necessário.

Em relação ao tempo gasto para o diagnóstico final, ele é menor no M3. Acreditamos que isso se deve ao fato de a lâmina já estar previamente marcada e, portanto, o escrutínio ser mais rápido, dirigido e com conhecimento de quais alterações devem ser procuradas.

Acreditamos que o desempenho dos métodos aplicados esteja relacionado a três fatores principais:

- número de vezes que o esfregaço é examinado: todas as lâminas foram examinadas no mínimo duas vezes nos métodos M2 e M3 (média de 2,2 vezes), enquanto a média no $\mathrm{M} 1$ foi de 1,04 vez;

- marcação de células: as células alteradas marcadas focam a atenção do examinador para determinado diagnóstico que deverá ser checado durante o desenvolvimento do escrutínio e, por isso, despertam maior atenção, concentração e rapidez desses examinadores;

- capacidade de detectar deficiências, diferenças e/ou dificuldades dos profissionais em relação à interpretação diagnóstica, induzindo-os à participação em programas de educação continuada: tanto o método $\mathrm{M} 2$ como o M3 são apropriados, e essa condição é apontada na literatura em relação ao $\mathrm{M} 2(\mathrm{RR})^{(2)}$.

Vários autores $(2,3,5,1012,19,22,31)$ defendem a revisão de todos os exames colpocitológicos após o escrutínio, para o controle interno da qualidade dos diagnósticos emitidos. Já o exame rápido pré-escrutínio, mas sem campo marcado, foi utilizado por um autor ${ }^{(29)}$ e esse relata bom resultado no controle da qualidade do diagnóstico, salientando ainda, que é possível que se faça a identificação de uma lesão intra-epitelial escamosa de alto grau num exame de 60 segundos. Por outro lado, outro autor ${ }^{(11)}$, após testar esse método de exame pré-escrutínio rápido, questiona a sua validade em relação ao controle de diagnóstico, entretanto reforça que são necessárias novas avaliações do método em outros serviços de citopatologia, para uma conclusão definitiva.

Sob o ponto de vista do marketing, o M3 (VR) com campo marcado, agregou valor ao serviço prestado, tornando-o um bom serviço ou produto. Esse valor agregado pode ser percebido pelos clientes externos/médicos, uma vez que eles têm a percepção de que ocorre concordância maior entre o diagnóstico da citologia convencional e o da colposcopia e/ou biópsia.

Conseguir menor número de diagnósticos $\mathrm{FN}$ sem aumento do custo é alcançar maior eficácia operacional. No M3 não houve gasto maior por exame realizado, já que não foi necessário aumentar o número de profissionais da equipe examinadora, despendeu-se menos tempo por exame e foram realizadas reuniões de estudo semanais, que antes não aconteciam com freqüência.

Portanto, o M3 (VR) com campo marcado permitiu alcançar um diferencial competitivo no mercado local, sem aumentar o custo operacional, mas apenas melhorando o controle da qualidade dos diagnósticos emitidos. Isso deve ser explorado na promoção do serviço laboratorial, visto que a probabilidade maior de o diagnóstico emitido ser verdadeiro-negativo agrega valor percebido pelos clientes. Obtivemos uma boa relação custo/benefício, encantando os clientes fiéis e atraindo novos clientes. Clientes novos aumentam a produtividade e sem produtividade não haverá prosperidade ${ }^{(8)}$. Em resumo, a comunicação eficaz, aliada a um bom produto ou serviço acessível ao mercado, com preço competitivo, são ingredientes fundamentais para o sucesso(7).

\section{Conclusão}

Os resultados observados em cada um dos métodos permitem concluir que o M3 (VR) com campo marcado, de autoria da pesquisadora, foi o que apresentou menor quantidade de diagnósticos falso-negativos $(1,2 \%)$, sendo considerado o melhor dos três métodos testados para controlar a qualidade interna do diagnóstico colpocitológico. 


\section{Agradecimentos}

A todos os colaboradores do Serviço de Anatomia Patológica da FAMERP/Fundação Faculdade Regional de
Medicina de São José do Rio Preto (FUNFARME) e às consultoras em NBR ISO 9001: 2000, as doutoras Maria Elisabete Mendes e Vera Lúcia P. Castilho.

\section{Referências}

I.ALVES,V.A.F.et al. Programa de controle de qualidade em citologia ginecológica do Instituto Adolfo Lutz: estratégias e análise crítica dos resultados de sua implantação piloto. RevAss Med Brasil, v. 37, p. 36-42, 1991.

2. AMARAL, R.G. Revisão rápida de esfregaços cervicais como método de garantia interna de qualidade.J Bras Patol e Med Lab, v. 39, p. I5I-5, 2003.

3.ARBYN, M.;SCHENCK, U. Detection of false negative pap smears by rapid reviewing. Acta Cytol, v. 44, p. 949-57, 2000

4. BAKER, R.W. The characteristics of false negative cervical smears: implications for the UK cervical cancer screening programmer.J Clin Pathol, v. 52, p. 358-62, 1999.

5. BAKER, A.; MELCHER, D. Rapid cervical cytology screening. Cytopathol, v. 2, p. 299-301, 1991.

6. BERKELEY,A.S.Advanced squamous cell carcinoma of the cervix with recent normal Papanicolaou tests. Lancet, v. 2, 375-6, 1980.

7. CAMARINHA, D. Comunicação eficaz: ferramenta de sucesso no laboratório. J SBAC, v. 18, p. 14, 2003.

8. CAPRONI, R. Como atrair e reter clientes. Disponível em: http:// www.caproni.com.br. Acesso em 18 set. 2004.

9. CARDIN,V. Replies to questions on quality assurance measures in cytopathology. Acta Cytol, v. 32, p. 915-9, 1988.

I0. DIEHL, A.R.S.; PROLLA, J.C. Rapid rescreening of cervical smears for internal quality control. Acta Cytol, v. 42, p. 949 53, 1998.

I I. FARAKER, C. Rapid screening in cervical cytology: a simple method with a big impact. Cytopathoil, v. I5, p. I70, 2004.

I2. FARRELL, D.J. Rapid screening of cervical smears as a method of internal quality control: for how long should we rescreen? Acta Cytol, v. 4I, p. 25 I-60, 1997.

I 3. GAY, J.D.; DONALDSON, L.D.; GOELLNER, J.R. False-negative results in cervical cytology studies. Acta Cytol, v. 29, p. I0436, 1985.

14. GILL, G.W. Pap smear risk management by process control. Cancer Cytopath, v. 8I , p. 198-21 I, 1997.

15. GONÇALVES, W.J.; GIRÃO, M.J.B.C.; BORTOLETTO, C.C.R. Rastreamento e diagnóstico precoce das neoplasias malignas genitais: perspectivas do arsenal propedêutico. J Bras Med, v. 69, p. 14-23, 1995.

16. GUIMARÃES, E.M.; SILVA, A.M. Erros em citopatologia ginecológica: por que ocorrem? J Bras Ginec, v. I05, p. $397-$ 404, 1995.

17. HUTCHINSON, M.L. Assessing the coats and benefits of alternative rescreening strategies. Acta Cythol, v. 40, p. 4-8, 1996.

18. KOSS, L.G.The Papanicolaou test for cervical cancer detection: a triumph and a tragedy. JAMA, v. 26, p. 737-43, 1989.

19. LEMAY, C.; MEISELS, A. 100\% rapid (partial) rescreening for quality assurance. Acta Cytol, v. 43, p. 86-9, 1999.

20. LORETO, C.D.I. et al. Garantia de qualidade em citopatologia: aspectos da correlação cito-histopatológica. Rev Ass Mod Brasil, v. 43,p. 195-8, 1997.

21. MARTIN, P.L. How preventable is invasive cervical cancer? A community study of preventable factors. Am J Obstet Gynecol, v. II3, p. 54I-8, 1972.

22. MELAMED, M.R. Reescrening for quality control in cytology. Acta Cytol, v. 40, p. 12-3, 1996.

23. MELAMED, M.R.; FLEHINGER, B.J. Reevaluation of quality assurance in the cytology laboratory. Acta Cytol, v. 36, p. $46 \mid-5,1992$

24. MINISTÉRIO DA SAÚDE. Programa Viva Mulher, 2002. Disponível em: <http://www.inca.gov.br/prevencao/ programas/viva_mulher>. Acesso em: 23 abr. 2003.

25. MITCHELL, H.; MEDLEY, G.; DRAKE, M. Quality control measures for cervical cytology laboratories. Acta Cytol, v. 32, p. 28892, 1988

26. NARYSHKINS, S. The false-negative fraction for Papanicolaou smears: how often are "abnormal" smears not detected by a "standard" screening cytologist? Arch Pathol Labor Med, v. 12, p. 270-2, 1997.

27. NORELL, N. D. False-negative cytology rates in patients in whom invasive cervical cancer subsequently developed. Obstet Gynecol, v. 60, p. 4I-5, 1982.

28. PATERSON, M.E.L. Cervical smears histories of 500 women with invasive cervical câncer in Yorkhire. Br Med J, v. 289, p. 896-8, 1984.

29. PLACIDI, A. et al. Rapid pre-screening of Pap smears in quality control: an Italian experience. Cytopatol, v. 15, p. 121, 2004.

30. RENSHAW, A.A. Analysis of error in calculating the falsenegative rate in the interpretation of cervical smears. Cancer Cytopathol, v. 8I, p. 264-7I, 1997.

31. RENSHAW,A.A. False negative rate of cervical cytologic smear screening as determined by rapid rescreening. Acta Cytol, v. 43, p. 344-50, 1998.

32. RYLANDER, E. Negative smears in women developing invasive cervical cancer. Acta Obstet Gynecol Scand, v. 56, p. I I5-8, 1977. 
33. SHIRATA, N.K.; PEREIRA, S.M.M.; CAVALIERI, M.J. Celularidade dos esfregaços cervicovaginais: importância em programas de garantia da qualidade. J Bras Ginec, v. 108, p. 63-6, 1998.

34.TRAVERS, H. Quality assurance indicators in anatomic pathology. Arch Pathol Lab Med, v. I I4, p. I |49-56, 1990.
35. VAN DER GRAAF, Y. Screening errors in cervical cytology screening. Acta Cytol, v. 3 I , p. 434-8, 1987.

36. WALKER, E.M. A retrospective review of cervical cytology in women developing invasive squamous cell carcinoma. $\mathrm{Br} J$ Obstet Gynecol, v. 90, p. 1087-91, 1983. 